\title{
IGF-IR in patients with advanced colorectal cancer in correlation with certain clinico-morphological factors: Initial report
}

\author{
ADAM KUKLINSKI $^{1}$, ZBIGNIEW KAMOCKI $^{1}$, MARIUSZ KODA ${ }^{2}$, ZDZISLAW PIOTROWSKI $^{1}$, \\ STANISLAW SULKOWSKI ${ }^{2}$, RYSZARD LESNIEWICZ ${ }^{3}$, KRYSTYNA PAWLAK $^{4}$, \\ PIOTR MYSLIWIEC ${ }^{1}$ and BOGUSLAW KEDRA ${ }^{1}$ \\ ${ }^{1}$ Second Department of General and Gastroenterological Surgery; Departments of ${ }^{2}$ General Pathomorphology, \\ ${ }^{3}$ Gynaecological Endocrinology and ${ }^{4}$ Monitored Pharmacotherapy, Medical University of Bialystok, Bialystok, Poland
}

Received March 17, 2011; Accepted August 3, 2011

DOI: $10.3892 / \mathrm{ol} .2011 .396$

\begin{abstract}
The insulin-like growth factor (IGF) system comprises two types of peptides (IGF-I and IGF-II), two types of receptors (IGF-IR and IGF-IIR) and six IGF-binding proteins (BP). This system is mainly responsible for the growth and division of cells in the body, regulation of the cell cycle and prevention of apoptosis. The expression of IGF-IR was assessed in the cells of resected primary colorectal tumours in 88 patients (age, 36-87 years; mean 64.78; males, 48 and females, 40) treated surgically at the Second Department of General and Gastroenterological Surgery, Medical University of Bialystok, Poland, in relation to various clinico-morphological factors. The post-operative material was analysed to find the histological type, location of lesions, lymph node involvement staging, distant metastases (pTNM classification), staging in Dukes' classification and the histopathological differentiation grade. The expression of IGF-IR in colorectal cancer cells was assessed using an immunohistochemical method. The findings were subjected to statistical analysis (Chi-square test, multivariation test and Mann-Whitney U test). A positive IGF-IR expression (in at least $10 \%$ of cancer cells) was observed in 44 patients. The mean immunoreactive cell count for IGF-IR in all of the tumours studied was $30.79 \%$. The current study showed no correlation of IGF-IR expression in colorectal cancer cells with characteristics such as age and gender of patients, tumour location, type, histological differentiation or histopathological advancement. Immunohistological determination of IGF-IR expression in advanced colorectal cancer cells revealed controversial scores. Evaluation should be confirmed by using other methods and enhanced to include adenomas and early colorectal cancers.
\end{abstract}

Correspondence to: Dr Adam Kuklinski, Second Department of General and Gastroenterological Surgery, Medical University of Bialystok, Sklodowska-Curie St 24A, 15-276 Bialystok, Poland E-mail: akuklinski@wp.pl

Key words: colorectal cancer, clinico-morphological factors, IGF-IR

\section{Introduction}

Insulin-like growth factors, IGF-I and IGF-II, together with their receptors, IGF-IR and IGF-IIR, and IGF-BP form a coherent system that is responsible for the growth and division of cells in the body (1). The system of insulin-like growth factors includes two receptors: type I, which transmits signals through tyrosine kinase, and type II, which is identical to the mannose-6-phosphate receptor that does not transmit signals but inhibits the auto- and paracrine functions of IGF-II through its uptake and internalisation from the plasma (2).

The IGF-IR receptor is a heterotetramer $(2 \alpha 2 \beta)$. The $\alpha$ subunits contain an IGF-I binding site, whereas the $\beta$ subunits start the process of phosphorylation and synthesis of intracellular proteins (1). The activated IGF-IR regulates the cell proliferation processes by transmitting division signals, protecting cells against apoptosis, regulating adhesion processes or inducing growth and differentiation of cells (3-6). Rouyer-Fessard et al found IGF-IR expression in normal colorectal mucosa (7). When overexpressed, IGF-IR behaves as a cell oncogene. The presence or absence of this receptor affects transformation of various viral and cellular oncogenes (3). A higher density of IGF-IR has been noted in carcinomas of the colon, ovary, breast, thyroid and endometrium, Wilms' tumours and gliomas $(1,8,9)$.

IGF-I and IGF-II acting on IGF-IR in colorectal cancer prevent apoptosis, enhance cell proliferation and induce the expression of vascular endothelial growth factor (VEGF). Results of experiments conducted on mice showed that IGF-IR, through VEGF induction, accelerates tumour growth and the formation of metastases $(5,10)$. However, the role of IGF-IR in cancer cells of the colon has yet to be fully elucidated. IGF-IR is frequently overexpressed in human colorectal cells. IGF-IR blockage results in the inhibition of growth and angiogenesis of colorectal carcinoma. A decrease in IGF-IR expression causes much apoptosis of cancer cells in vivo and in vitro. In experimental animal studies, IGF-IR hypoexpression is manifested as the inhibition of tumour and metastasis formation (6).

Taking the above into consideration, this study aimed to assess the expression of IGF-IR in colorectal cancer cells in correlation with certain clinico-morphological factors. 


\section{Patients and methods}

Patients. The study included 88 patients with primary colorectal carcinoma treated surgically at the Second Department of General and Gastroenterological Surgery, Medical University of Bialystok, Poland, in the years 1998-2003. The study group comprised 48 (54.6\%) males and 40 (45.4\%) females with an average age of 64.78 years (range 36-87). Twenty-eight $(31.8 \%)$ patients were $<60$ years of age, and the remaining $60(68.2 \%)$ patients were $>60$ years of age. As regards tumour location, the patients were divided into two groups. The first group included 43 (48.9\%) patients with rectal carcinoma. The patients with carcinoma elsewhere in of the colon $(45 ; 51.1 \%)$ constituted the other group. Patients underwent scheduled surgery. Eighteen (20.5\%) patients had abdominoperineal excision of the rectum by the Miles method. In 27 (30.7\%) patients, low anterior resection of the rectum was performed with an end-to-end anastomosis. Hartmann's operation was carried out in 19 (21.6\%) patients. Right-side hemicolectomy was performed in $17(19.3 \%)$ patients, whereas left-side hemicolectomy was performed in $4(4.5 \%)$ patients. A further $3(3.4 \%)$ patients underwent segmental excision of the transverse colon.

Routine histopathological investigations were performed to analyse tumor node metastasis (TNM) and Dukes' staging of anatomo-clinical advancement, histological type and malignancy grade $(\mathrm{G})$.

Lesions in the pT3 and pT4 stages were found to predominate in the TNM classification. There were no $\mathrm{pT} 1$ patients. The pT2 group included $8(9.1 \%)$ individuals. Sixty-one (69.3\%) patients had tumours in the pT3 stage and $19(21.6 \%)$ in the pT4 stage. Forty (45.4\%) patients had no local lymph node involvement (group N0). Group N1 comprised 24 (27.3\%) patients; group N2, $22(25 \%)$ patients and group N3 had a further $2(2.3 \%)$ patients. Liver metastases (stage M1) were observed in only $6(6.8 \%)$ patients. The remaining $82(93.2 \%)$ patients had no distant metastases (stage M0). Advancement of the neoplastic process was estimated in the Dukes' classification as modified by Astler-Coller. There were no patients in group A, 5 (5.7\%) patients in group B1, 34 (38.6\%) in group B2, $13(14.8 \%)$ in group $\mathrm{C} 1,30(34.1 \%)$ in group $\mathrm{C} 2$ and $6(6.8 \%)$ in group $\mathrm{D}$.

Any lesions were verified by histopathological examination. Adenocarcinoma was found in 68 (77.3\%) cases, mucogenic adenocarcinoma in $7(8 \%)$ and partly mucogenic adenocarcinoma in $10(11.4 \%)$ patients. Poorly differentiated adenocarcinoma (1.1\%), ulcerative adenocarcinoma (1.1\%) and mucocellular carcinoma (1.1\%) were only identified in single cases.

In the study group, highly differentiated carcinomas (G1) were not detected. Moderate differentiation (G2) was observed in $57(64.8 \%)$ patients, whereas low differentiation (G3) was found in the remaining 31 (35.2\%) patients.

Materials. Two specimens from each tumour were stained using an immunohistochemical method of evaluation. A standard avidin-biotin immunoperoxidase method (ABC Staining System, Santa Cruz Biotechnology, Santa Cruz, CA, USA) was used for the detection of IGF-IR expression. In all cases, specimens were obtained from the main mass of the tumour.
The specimens were fixed in $40 \mathrm{~g} / \mathrm{l}$ formaldehyde, embedded in paraffin and cut into $4-\mu \mathrm{m}$ sections. The sections were dewaxed in three changes of xylene and hydrated through an alcohol series of a decreasing concentration. The sections were heated for $3 \mathrm{~min}$ in citrate buffer $(10 \mathrm{mmol} / 1, \mathrm{pH} 6.0)$ in a pressure cooker to expose the antigen. Endogenous peroxidase activity was blocked by incubating the sections in a 3\% hydrogen peroxide solution in methanol for $5 \mathrm{~min}$. The slides were then washed 3 times in phosphate-buffered saline (PBS) and incubated in normal horse serum for $5 \mathrm{~min}$ to reduce non-specific antibody binding. Following washing with PBS, the slides were incubated for $24 \mathrm{~h}$ at $4^{\circ} \mathrm{C}$ with monoclonal antibody (Anti-IGF-IR, $\beta$ subunit, C-terminal clone CT-3) (H-60, Santa Cruz Biotechnology) at a dilution of 1:100 for all slides. The antigen-antibody complex was visualised by DAB chromogen (3'3-diamonobenzidine, Dako, Denmark). Following rinsing in distilled water, the sections were stained with hematoxylin, and following dehydration with an alcohol series of an increasing concentration the sections were mounted on a Canadian balsam. A light microscope was used for the analysis of the immunohistochemical reactions in colorectal carcinoma cases. The expression of IGF-IR was analysed in 10 various fields of vision, in which the mean percentage of the immunohistochemically positive cancer cells was determined (positive when $>10 \%$ of carcinoma cells were IGF-IR-positive; negative when there was no reaction or $\leq 10 \%$ of cells were positive). Control immunohistochemical staining was performed; the positive control comprised IGF-IR-positive colorectal cancer specimens, in the negative control primary antibodies were omitted in the staining procedure. The negative control did not exhibit any specific immunostaining.

Statistical analysis. The results were subjected to statistical analysis using the Chi-square test, multivariate analysis and the Mann-Whitney U test. Differences were considered statistically significant at $p<0.05$. Statistical analysis was performed using the statistical package SPSS 8.0 PL.

\section{Results}

Immunoreactivity. IGF-IR expression was assessed in 88 colorectal tumours. In $44(50 \%)$ of the examined colorectal tumours an immunohistochemical reaction to IGF-IR was evident. The presence of an immunohistochemical reaction in at least $10 \%$ of cancer cells was referred to as a positive reaction. In all the tumours, the mean IGF-IR immunoreactive cell count was found to be $30.79 \%$. The Mann-Whitney U test and multivariate analysis did not yield statistical differences.

Correlation between IGF-IR expression and patient characteristics. The Chi-square test was used to investigate the relationship of IGF-IR expression with characteristics such as patient age and gender, tumour location, histological type, clinicopathological advancement in Dukes' classification, pTNM and histological differentiation. Due to the data distribution, the patients were divided into three groups: i) negatively immunoreactive against IGF-IR (IGF-IR <10\%), ii) moderately immunoreactive against IGF-IR (IGF-IR 11-50\%), iii) highly immunoreactive against IGF-IR (IGF-IR $>50 \%$ ). 


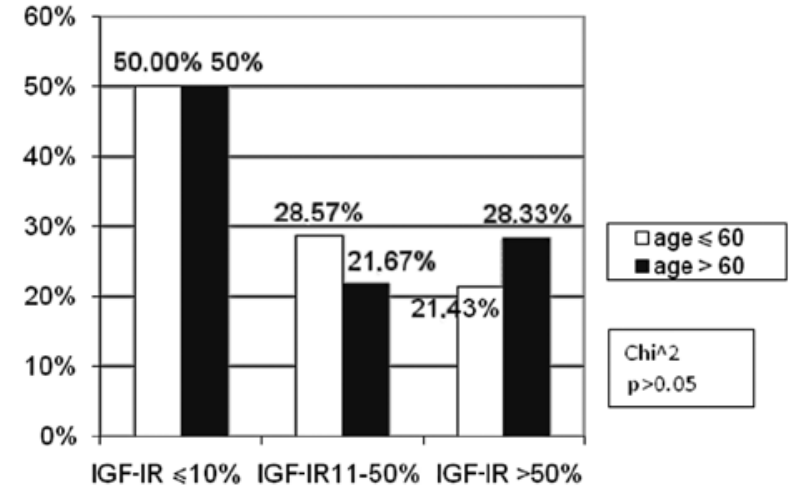

Figure 1. Correlation between the percentage of immunoreactive cells for IGF-IR and age.

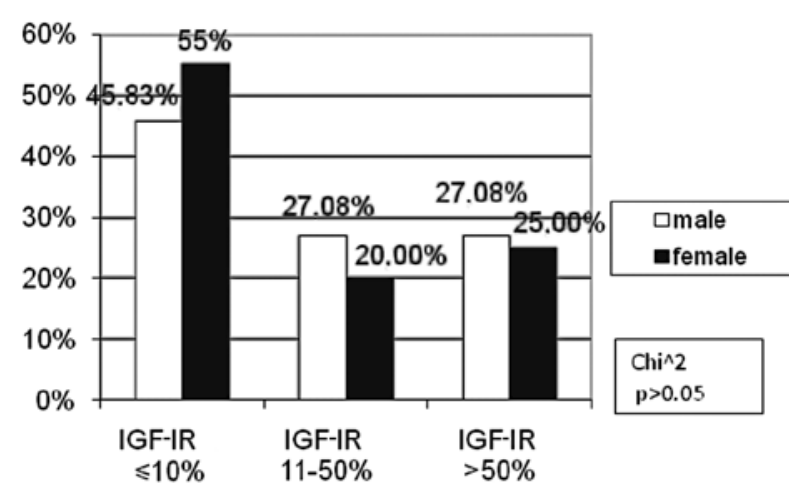

Figure 2. Correlation between the percentage of immunoreactive cells for IGF-IR and gender.

The patients constituted two age groups: $<60$ and $>60$. The number of patients at these ages did not differ between the subgroups of patients with negative, moderate and high IGF-IR expression (Fig. 1).

The percentage of IGF-IR negative male patients (45.83\%) was markedly lower in comparison to females (55\%). Conversely, in the moderate IGF-IR subgroup, there were more male $(27.08 \%)$ than female (20\%) patients. These differences were not statistically significant ( $>00.05$, Fig. 2).

According to tumour location, the patients were divided into rectal carcinoma and colon carcinoma subgroups. No difference was observed in the immunohistochemical reaction to IGF-IR compared to the colorectal cancer location (Fig. 3).

Only adenocarcinomas and mucogenic adenocarcinomas were considered for the analysis of histopathological type due to the small number of remaining tumours. In the subgroup exhibiting a high IGF-IR expression, more patients had mucogenic adenocarcinoma (33.33\%) than those with adenocarcinoma (24.29\%), the difference being statistically insignificant ( $p>0.05$, Fig. 4).

In the TNM classification, with regard to the $\mathrm{pT}$ feature, the patients were divided into two groups: pT2 and pT3/pT4. No patient was included in the pT1 subgroup. For the pN and pM features, two groups were distinguished: patients with and without metastases (pN0, pM0 and pN1 and pM1, respectively). As for the tumour size, in the high IGF-IR expression subgroup

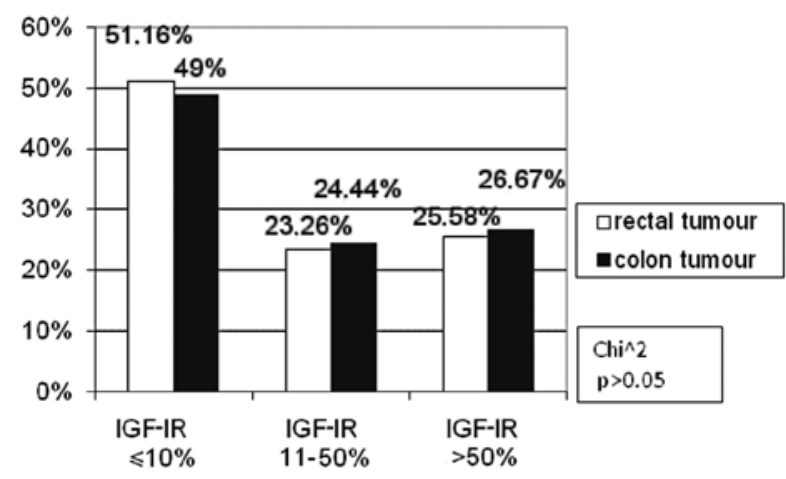

Figure 3. Correlation between the percentage of immunoreactive cells for IGF-IR and colorectal cancer location.

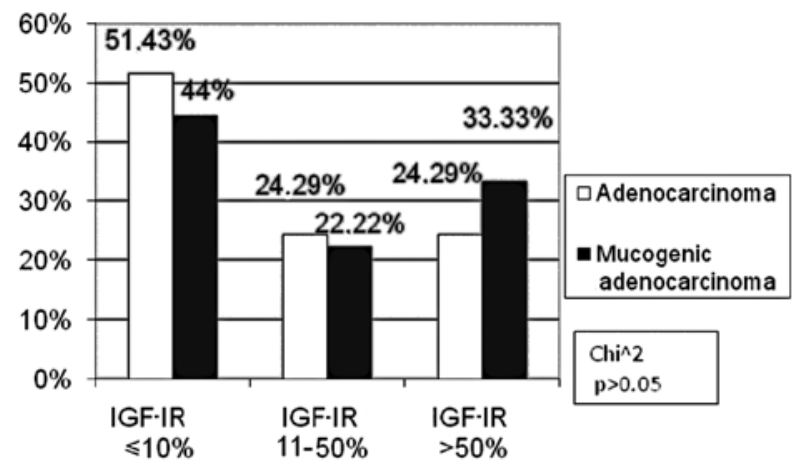

Figure 4. Correlation between the percentage of immunoreactive cells for IGF-IR and the histopathological type of tumour.

the percentage of pT3/pT4 (25.32\%) patients was statistically insignificantly lower as compared to pT2 (33.33\%) patients. In the other subgroups, the percentage of patients was similar (Fig. 5). There were fewer patients with lymph node involvement than those without metastases in the negative IGF-IR expression subgroup (46 vs. 55\%). However, in the low IGF-IR expression subgroup, the number of patients with lymph node involvement was higher as compared to the metastasis-free cases (29.17 vs. $17.5 \%)$. However, the difference was not statistically significant ( $p>0.05$, Fig. 6). The percentage of patients with distant metastases (pM1) was markedly higher $(60.00 \%)$ as compared to the metastasis-free subjects (pM0) (48.78\%) in the negative IGF-IR expression subgroup, the difference being statistically insignificant ( $>0.05)$. No differences were found in the remaining groups (Fig. 7).

According to Dukes' classification, two subgroups were distinguished: Dukes' B, and Dukes' C and D. No Dukes' A patients were found. This division was determined by the presence or absence of lymph node involvement. The percentage of Dukes' C and D patients was markedly higher (28.57\%) than Dukes' B (17.95\%) in the low IGF-IR expression subgroup; however, there was no statistical significance ( $p>0.05)$. Similar numbers of Dukes' B, C and D patients were noted in the other subgroups of IGF-IR expression (Fig. 8).

The percentage of patients with a moderate histological differentiation grade was statistically insignificantly higher (G2: $53.57 \%$ ) as compared to those with a low grade 


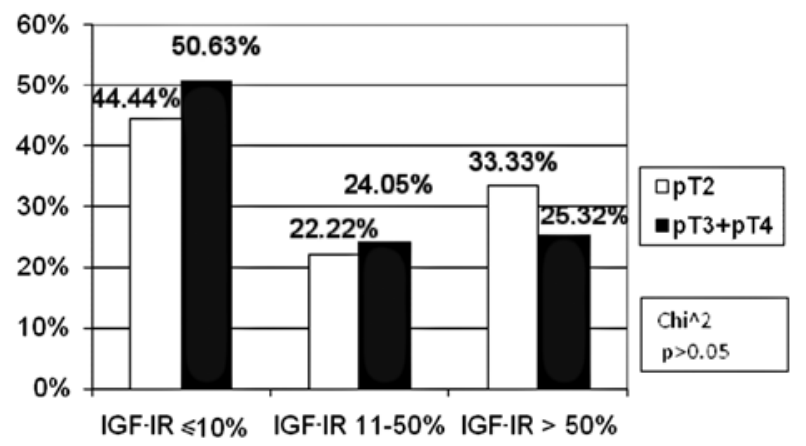

Figure 5. Correlation between the percentage of immunoreactive cells for IGF-IR and tumour size (pT).

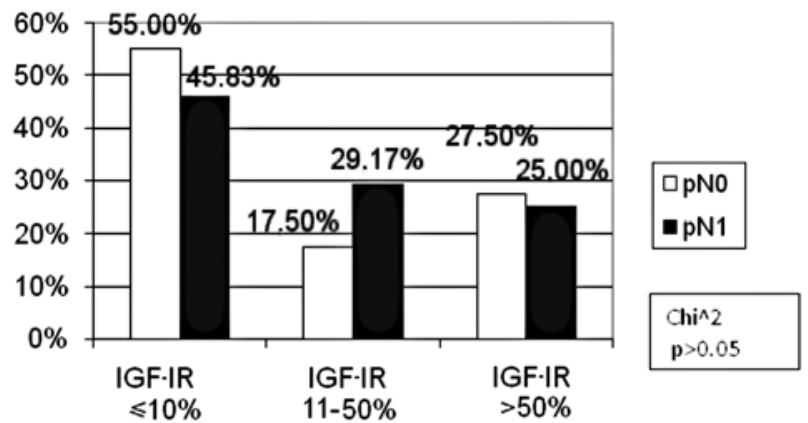

Figure 6. Correlation between the percentage of immunoreactive cells for IGF-IR and lymph node metastases (pN).

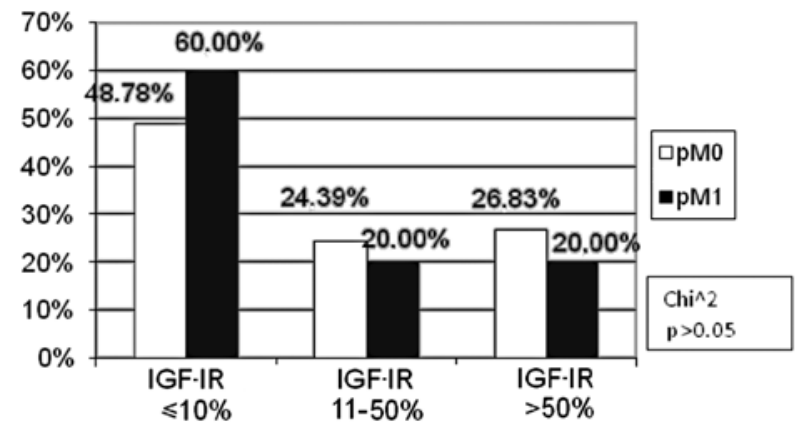

Figure 7. Correlation between the percentage of immunoreactive cells for IGF-IR and distant metastases (pM).

(G3: 41.94\%) in the negative IGF-IR expression subgroup $(\mathrm{p}>0.05)$. No difference was found in the percentage of moderate and low-differentiated tumours in the remaining subgroups of IGF-IR expression (Fig. 9).

\section{Discussion}

In this study, a positive IGF-IR expression in $50 \%$ of the colorectal carcinomas examined was observed. The mean percentage of IGF-IR immunoreactive cells was $30.79 \%$ in the whole study group. Weber et al described a positive immunohistochemical reaction for IGF-IR in the cells of $91 \%$ of the colorectal cancers studied (11). Hakam et al also observed a

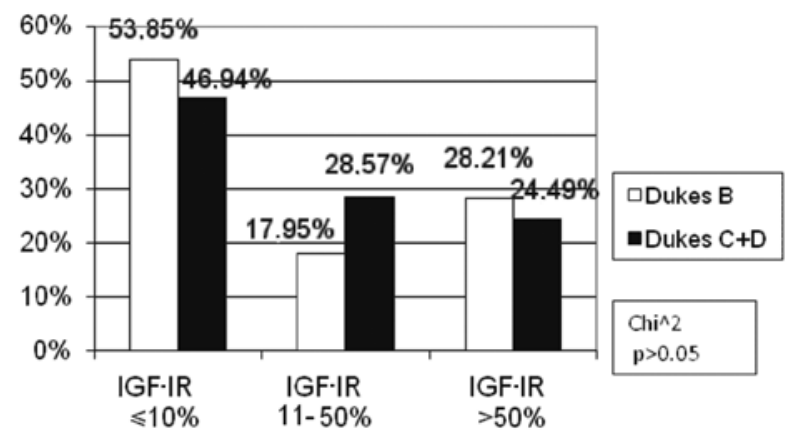

Figure 8. Correlation between the percentage of immunoreactive cells for IGF-IR with clinical and morphological advancement in Dukes' classification.

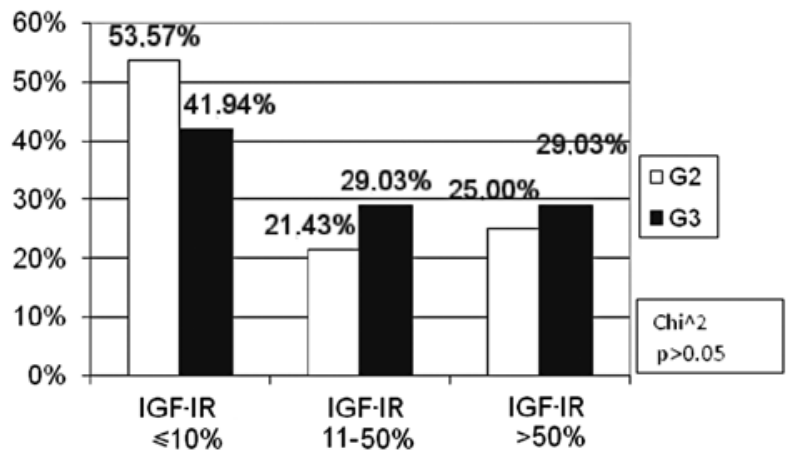

Figure 9. Correlation between the percentage of immunoreactive cells for IGF-IR and histological differentiation.

positive immunohistochemical reaction in $96 \%$ of the tumours examined. The authors found the enhanced IGF-IR expression to correlate with the histopathological differentiation grade and with the anatomopathological advancement of colorectal cancer. According to these investigators, a higher expression of IGF-IR may promote the formation of metastases (12). On the other hand, neither Adenis nor Zenilman, who studied the expression of IGF-IR mRNA in colorectal carcinoma patients, observed an elevated IGF-IR expression in colorectal cancer cells $(13,14)$. Nosho et al found an increased IGF-IR expression in $37.8 \%$ of the colorectal carcinomas studied (15). The findings reported by Pollak et al explain the discrepancies between the present results and the majority of the literature data (16). These authors have shown that a lower IGF-IR expression may be observed in high-grade colorectal carcinomas, including prostate carcinoma (10). In the present study group, almost $20 \%$ of patients were classified as pT4 according to the pTNM classification. A lower percentage of pT3 and pT4 as compared to pT2 patients in the high IGF-IR expression subgroup were noted.

No statistically significant correlation was found between the increased IGF-IR expression in colorectal cancer cells with characteristics such as patient age and gender, tumour location, histological type, advancement or lymph node involvement. However, an increased number of immuno-reactive cells for IGF-IR in low-differentiated colorectal cancers was observed, the differences being statistically insignificant. Similarly, Nosho et al observed no correlation of IGF-IR expression 
in colorectal cancer cells with clinical and pathomorphological factors (15). No other data concerning the correlation of IGF-IR expression with clinical and pathomorphological factors were detected. Results of the present study suggest that the determination of IGF-IR expression in advanced colorectal cancer may have a limited prognostic and diagnostic value.

This study did not include patients with adenomas and only a few of the patients had early cancer. Teramukai et al assert that the plasma concentration of IGF-I may change during the carcinoma sequence of colorectal cancer (17). Estimation of the changes in IGF-IR expression in normal mucous, adenomas and early cancers of the large bowel appear to be justified.

Assessment of the number of cells with a positive immunohistochemical reaction for IGF-IR may be applied when quali-fying patients for therapy. As shown in certain publications, blockage of IGF-IR effectively increases cancer cell apoptosis (18). Anticancer therapy based on the action of one of the GH-IGF-IGFR axis links leads to high expectations, particularly for colorectal carcinoma (5). A number of studies have concluded that IGF-IR is the most promising target in anticancer therapy $(16,18-22)$.

The majority of data concerning IGFs are based on in vitro studies. Studies in vivo are rare, and frequently provide contradictory and unclear evidence. Broader studies on IGFs are required (23), as they may aid in the search for an early detection mode and facilitate the design of effective anti-colorectal cancer therapy.

Based on the study findings, the following conclusions have been formulated: i) no correlation was found between IGF-IR expression and the clinical and pathomorphological factors studied, ii) a controversial low expression of IGF-IR in the advanced colorectal cancer cells studied delimits the usefulness of immunohistochemical assessment of IGF-IR in the prognosis of the course of the neoplastic process and simultaneously stimulates further investigations.

\section{References}

1. Mauro L and Surmacz E: IGF-I receptor, cell-cell adhesion, tumor development and progression. J Mol Histol 35: 247-253, 2004.

2. Grimberg A and Cohen P: Role of insulin-like growth factor and their binding proteins in growth control and carcinogenesis. J Cell Physiol 183: 1-9, 2000

3. Adams TE and Epa VC: Structure and function of the type I insulin-like growth factor receptor. Cell Moll Life Sci 57: 1050-1093, 2000.

4. Baserga R: The IGF-I receptor in cancer research. Exp Cell Res 253: 1-6, 1999.

5. Reinmuth N, Fan F, Liu W, Parikh AA and Stoeltzing O: Impact of insulin-like growth factor receptor-I function on angiogenesis, growth and metastasis of colon cancer. Lab Invest 82: 1377-1389, 2002.
6. Valentinis B and Baserga R: IGF I receptor signaling in transformation and differentiation. J Clin Pahol 54: 133-137, 2001.

7. Rouyer-Fessard C, Gammeltoft S and Laburthe M: Expression of two types of receptor for insulin-like growth factors in human colonic epithelium. Gastroenterol 98: 703-707, 1990.

8. Renehan AG, Painter JE, O'Halloran D, Atkin WS, Potten CS, O'Dwyer ST and Shalet SM: Circulating insulin-like growth factor II and colorectal adenomas. J Clin Endocrinol Metab 85: 3402-3408, 2000.

9. Wu Y, Yakar S, Zhao L, Hennighausen L and LeRoith D: Circulating insulin-like growth factor-I levels regulate colon cancer growth and metastasis. Cancer Res 15: 1030-1035, 2002.

10. Reinmuth N, Liu W, Fan F, Jung YD and Ahmed SA: Blockade of insulin-like growth factor I receptor function inhibits growth and angiogenesis of colon cancer. Clin Cancer Res 8: 3259-3269, 2002.

11. Weber MM, Fottner C, Liu SB, Jung MC, Engelhardt D and Baretton GB: Overexpression of the insulin-like growth factor I receptor in human colon carcinomas. Cancer 95: 2086-2095, 2002.

12. Hakam A, Yeatman TJ, Lu L, Mora L, Marcet G, Nicosia SV, Karl RC and Coppola D: Expression of insulin-like growth factor-1 receptor in human colorectal cancer. Hum Patol 30: 1128-1133, 1999.

13. Adenis A, Peyrat JP, Hecquet B, Delobelle A, Depadt G, Quandalle P, Bonneterre J and Demaille A: Type I insulin-like growth factor receptors in human colorectal cancer. Eur J Cancer 31: 50-55, 1995.

14. Zenilman ME and Graham W: Insulin-like growth factor I receptor messenger RNA in the colon is unchanged during neoplasia. Cancer Invest 15: 1-7, 1997.

15. Nosho K, Yamamoto H, Taniguchi H, Adachi Y, Yoshida $Y$, Arimura $\mathrm{Y}$, Endo $\mathrm{T}$, Hinoda $\mathrm{Y}$ and Imai $\mathrm{K}$ : Interplay of insulin-like growth factor-II, insulin-like growth factor-I, insulin-like growth factor-I receptor, $\mathrm{COX}-2$, and matrix metalloproteinase-7, play key roles in the early stage of colorectal carcinogenesis. Clinical Cancer Res 10: 7950-7957, 2004.

16. Pollak MN, Perdue JF, Margolese RG, Baer K and Richard M: Presence of somatomedin receptors on primary human breast and colon carcinomas. Cancer Lett 38: 223-230, 1987.

17. Teramukai S, Rohan T, Lee KY, Eguchi H, Oda T and Kono S: Insulin-like growth factor (IGF)-I, IGF-binding protein-3 and colorectal adenomas in Japanese men. Jpn J Cancer Res 93: 1187-1194, 2002.

18. Adachi Y, Lee CT, Coffee K, Yamagata N, Ohm JE, Park KH, Dikov MM, Nadaf SR, Arteaga CL and Carbone DP: Effects of genetic blockade of the insulin-like growth factor receptor in human colon cancer cell lines. Gastroenterology 123: 1191-1204, 2002.

19. Hassan AB and Macaulay VM: The insulin-like growth factor system as a therapeutic target in colorectal cancer. Ann Oncol 13: 349-356, 2002.

20. Moschos SJ and Mantzoros CS: The role of the IGF system in cancer: from basic to clinical studies and clinical applications. Oncology 63: 317-332, 2002.

21. Khandwala HM, McCutcheon IE, Flyvbjerg A and Friend KE: The effects of insulin-like growth factors on tumorigenesis and neoplastic growth. Endocr Rev 21: 215-244, 2000.

22. Sandhu MS, Dunger DB and Giovannucci EL: Insulin, insulin-like growth factor-I (IGF-I), IGF binding proteins, their biologic interactions, and colorectal cancer. J Natl Cancer Inst 94: 972-980, 2002.

23. Durai R, Yang W, Gupta S, Seifalian AM and Winslet MC: The role of the insulin-like growth factor system in colorectal cancer: review of current knowledge. Int $\mathbf{J}$ Colorectal Dis May 20: 203-220, 2005 